\title{
THE INFLUENCE OF ALEXITHYMIA ON MEMORY FOR EMOTIONAL FACES AND REALISTIC SOCIAL INTERACTIONS
}

\author{
Nathan Ridout, Jade Smith and Holly Hawkins
}

Aston University, UK

Address for correspondence:

Dr Nathan Ridout

Department of Psychology,

School of Life \& Health Sciences,

Aston University,

Birmingham, B47ET

UK

Email: Dr Nathan Ridout n.ridout@aston.ac.uk 


\title{
THE INFLUENCE OF ALEXITHYMIA ON MEMORY FOR EMOTIONAL FACES AND REALISTIC SOCIAL INTERACTIONS
}

\begin{abstract}
High levels of alexithymia are typically associated with impaired memory for emotional, but not neutral words. We conducted two experimental studies to establish if this effect generalises to non-verbal socially relevant stimuli. Thirty-nine female undergraduates (Study 1) viewed faces with different expressions (neutral, angry, happy or sad) and 38 female students (Study 2) viewed videos of realistic social interactions (featuring anger, happiness, sadness or neutral affect). Participants were asked to identify the emotion portrayed and were subsequently given an intentional recognition memory test for the stimuli. They also completed self-report measures of alexithymia and mood (depression \& anxiety). In Study 1, memory for emotional (especially angry), but not neutral faces was negatively related to the 'difficulty describing feelings' facet of alexithymia. In Study 2, memory for emotional (particularly those featuring anger), but not neutral videos was negatively related to the 'difficulty identifying feelings' and 'externally oriented thinking' facets of alexithymia. In both studies, these memory deficits were independent of the effects of age and mood. Furthermore, the deficits appear to be most evident in the conscious recollection of the emotional stimuli. Our findings confirm that the memory deficit for emotional words in alexithymia generalises to important non-verbal socially relevant stimuli.
\end{abstract}

Key words: alexithymia; memory; facial-expressions; social-interactions; depression 


\section{Introduction}

Alexithymia (ALX) is a multifaceted construct that is associated with difficulties identifying and describing one's feelings, problems differentiating between bodily sensations (e.g. hunger) and affect, a lack of imagination or fantasies, and an externally oriented cognitive style (Taylor, Bagby \& Parker, 1997). The Toronto Alexithymia Scale (TAS-20; Bagby, Parker \& Taylor, 1994ab) is the most widely used self-report measure of alexithymia, which assesses the three core facets of the alexithymia construct, difficulty identifying feelings (DIF), difficulty describing feelings (DDF) and externally oriented thinking (EOT). DIF concerns the ability to identify one's feelings (e.g. "I am often confused about what emotion I am feeling”), DDF refers to the ability to put one's feelings into words (e.g. "It is difficult for me to find the right words for my feelings"), whereas EOT concerns a focus on external as opposed to internal aspects of a person's experience (e.g. "I prefer to speak to people about their daily activities rather than their emotions"). Scores on the three subscales are combined to provide an overall measure of alexithymia (total TAS-20 score). Using the recommended cut off (a total TAS-20 score of 62 or greater), Mason et al (2005) reported a prevalence rate of $17.92 \%$ for alexithymia in a sample of UK students. However, it should be noted that alexithymia scores tend to be higher in participants exhibiting symptoms of psychological distress, notably depression and anxiety (Honkalampi et al., 2000).

Suslow, Kersting and Arholt (2003) characterised alexithymia as a deficit in the cognitive processing of affect. In line with this conception, there is a growing body of evidence that alexithymia is associated with marked changes in memory function. For example, alexithymia appears to undermine the memory advantage for emotional material that has been well-established in the literature (see reviews in LaBar \& Cabeza, 2006; Talmi, 2013). Suslow et al. (2003) reported that memory for emotional words was negatively associated with scores on the DIF subscale of the TAS-20. Similarly, Luminet, Vermeulen, 
Demaret, Taylor and Bagby (2006) reported that participants exhibiting high levels of alexithymia (total TAS-20 score) recalled fewer positive and negative words in comparison to individuals with low levels of alexithymia. Notably, both of these studies demonstrated that memory for neutral words was unaffected by alexithymia. Luminet et al. (2006) utilised the Remember-know paradigm (Gardiner, 1988) and reported that the memory deficit for emotional words in high alexithymia scorers was only evident in the Remember and not the Know responses. Remember responses are where the participant consciously recollects seeing the item during encoding, including details of the context (e.g. what they were thinking at the time). Know responses, on the other hand, are where the participant only experiences a feeling of familiarity about the item, without a conscious recollection of the context in which it was experienced. With this in mind, the findings of Luminet al (2006) suggest that alexithymia is associated with impaired conscious recollection of emotional words. Vermeulen and Luminet (2009) extended this work by examining the influence of alexithymia on memory for specific categories of words denoting joy, anger, disgust and neutral affect. Their findings also demonstrated that memory for emotional, but not neutral words was negatively related to alexithymia (DIF scores) and this relationship was stronger for Remember than Know responses. It has been suggested that the recollective advantage for emotional material over neutral is due to the emotional material being encoded more distinctively than the neutral material (Ochsner, 2000). Further, it has been proposed that alexithymia is associated with problems in the symbolic representation of emotions (i.e. using words or images), thus it is argued that individuals exhibiting elevated levels of alexithymia have less elaborated/ integrated emotional schema (Luminet et al., 2006; Suslow et al, 2003). With this in mind, one potential explanation for impaired memory for emotional material in alexithymia is that valence may be less conceptually salient for individuals with high alexithymia. 
Vermeulen, Toussaint and Luminet (2010) confirmed the finding that alexithymia is associated with impaired memory for emotional words. However, this relationship varied as a function of the emotional context (manipulated using background music). In the context of angry music, memory for joy words was negatively associated with DIF and DDF scores, DDF scores were also negatively associated with memory for angry words. When the background music was happy, memory for joy words was positively associated with DIF scores and memory for angry words was negatively related to EOT scores, which strongly suggests that the relationships between alexithymia and memory for emotional stimuli are context dependent. One possible explanation is that the emotional context created by the music may have altered the relative salience of the different affective words. Interestingly, Meltzer and Nielson (2010) demonstrated that although individuals with high alexithymia (total TAS-20 score) recalled fewer negative words (e.g. 'hatred'), they recalled a greater number of illness-related words (e.g. 'pain'), which confirms that individuals with high alexithymia can remember emotional words when they are personally salient.

Although the memory deficit for emotional words in high alexithymia scorers would appear to be robust, some studies have failed to find any effect of alexithymia on memory function (Jacob \& Hautekeete, 1998; Lundh, Johnsson, Sundqvist, \& Olsson, 2002). This could plausibly due to variations in the experimental methods employed in the different studies. For example, Lundh et al. (2002) examined retrieval times on the autobiographical memory test (AMT; Williams and Broadbent, 1986), whereas studies reporting a memory deficit in alexithymia have tended to examine memory content. Furthermore, although studies have tended show intact memory for neutral material in high alexithymia scorers (Luminet et al, 2006; Suslow et al., 2003; Vermeulen \& Luminet, 2009), Correro, Paitel, Byers and Nielson (2019) provided evidence of impaired memory for neutral material in participants with elevated alexithymia scores. 
To date, work investigating the relationship between memory and alexithymia has focused on changes in memory for verbal material. A notable exception is a study conducted by DiStefano and Koven (2012) who examined the influence of alexithymia on intentional memory for non-emotional (neutral) faces and images of people in social scenes. They reported that individuals with high TAS-20 scores exhibited impaired memory for people in social scenes relative to low scorers. However, the two groups did not differ in their memory for neutral faces. This is important, as it confirms that the effects of alexithymia on memory are not confined to verbal stimuli. Given that there was an emotional dimension to the social images it could be argued that these findings are consistent with the memory deficit that has been observed in verbal material (i.e. impaired memory for emotional but not neutral material). However, as the social images differed from the neutral faces on several factors in addition to the emotional content (e.g. the images were more complex), it is difficult to draw firm conclusions from these data. It remains unclear if alexithymia would influence memory for individual faces featuring emotional expressions. Takahashi, Hirano and Gyoba (2015) examined short-term memory for emotional faces in alexithymia using a change detection paradigm. They demonstrated that alexithymia was associated with impaired visual shortterm memory for faces with happy, but not angry expressions. However, as their study examined short-term memory and did not include neutral faces as a comparison, it has yet to be determined if the pattern of long-term memory deficits exhibited by high alexithymia scorers generalise from verbal to non-verbal stimuli. Therefore, the aim of the current study was to investigate if variations in alexithymia influenced recognition memory for faces. Specifically, we examined if high levels of alexithymia were associated with reduced memory for emotional, but not neutral faces.

It is notable that alexithymia is associated with high levels of depression and anxiety (Honkalampi et al., 2000) factors that have been shown to influence memory for emotional 
material, including faces (Mayer, McCormick \& Strong, 1995; Noreen \& Ridout, 2010; Ridout et al., 2003; 2009ab). With this in mind, it is important to control for the presence of these mood variables when examining the influence of alexithymia on memory for faces. Similarly, as there is evidence of a relationship between age and alexithymia scores in student samples (Bagby, Parker \& Taylor, 1994) there is a need to control for the potential influence of this factor when analysing the current data.

\section{Study 1}

Overview and Predictions

The aim of Study 1 was to determine if alexithymia is associated with variations in memory for emotional and neutral faces. To this end, a group of female undergraduates was presented with a series of emotional and neutral faces and asked to identify the emotion portrayed by each face (a forced choice from happy, sad, angry or neutral). Following a 5minute filled delay, the faces were presented again, intermingled with a set of novel 'foils', and participants were invited to make 'old/ new' and 'remember/ know/ guess' judgements. They also completed the 20 -item Toronto Alexithymic Scale (TAS-20; Bagby, Parker, \& Taylor 1994) and the Hospital Anxiety and Depression Scale (HADS; Zigmond \& Snaith, 1983). Based on previous research (e.g. Grynberg et al., 2012), it was predicted that the percentage of facial expressions correctly labelled would be negatively related to scores on the subscales of TAS-20. In line with Vermeulen and Luminet (2009) and DiStefano and Koven (2012), it was expected that memory for faces would be negatively related to scores on the subscales of the TAS-20. However, it was expected that this relationship would only be evident for emotional and not neutral faces. Furthermore, in line with Vermeulen and Luminet (2009), it was expected that the relationship between alexithymia and memory for emotional faces would only be evident in the Remember responses and not in the Know 
responses. Importantly, these relationships were expected to be independent from the influence of age and mood (depression and anxiety).

\section{Method}

\section{Participants}

Thirty-nine ${ }^{1}$ female ${ }^{2}$ undergraduate students took part in the study in exchange for course credit. The mean age of the sample was 19.5 years $(\mathrm{SD}=1.1)$. Individuals with a history of psychiatric illness were requested not to take part in study. The study was approved by Aston University's Research Ethics Committee and full written informed consent was gained from all participants prior to taking part in the study.

\section{Measures and tasks}

The 20-item Toronto alexithymia scale (TAS-20; Bagby, Parker, \& Taylor 1994) is the most widely used measure of alexithymia, which has been shown to be both valid and reliable. For example, Parker, Taylor and Bagby (2003) reported an internal reliability coefficient of .86. Each item consists of a statement, e.g. "I am often confused about what emotion I am feeling”, which is then rated using a 5-point Likert scale ranging from 1 'Strongly disagree' to 5 'Strongly agree', thus the total score on the TAS-20 ranges between 20 and 100, with higher scores indicating more severe alexithymia. The items on the TAS-20 are split into three factors

\footnotetext{
${ }^{1}$ Based on the observed effect size reported in a previous study examining the influence of alexithymia on memory for faces (DiStefano \& Koven, 2012) a large effect size $\left(\mathrm{f}^{2}=.35\right)$ was expected. The results of a power calculation using $\mathrm{G}^{*}$ Power to determine the required sample size for a multiple regression to detect a significant change in $\mathrm{R}^{2}$ with 3 tested predictors and a total of 6 predictors revealed that a minimum of 36 participants would be required to detect a large effect size with a power of .8 and an alpha level of .05 . Therefore, this study was sufficiently powered.

${ }^{2}$ Five male participants were also recruited. However, given that males and females differ in face processing (Sullivan et al., 2015) and alexithymia (Levant et al., 2009), and that there was an insufficient number of males to allow for a reliable group comparison we chose to exclude these participants from the analysis of the data from Study 1.
} 
that relate to different aspects of alexithymia: 'difficulty identifying feelings' (DIF), 'difficulty describing feelings' (DDF) and 'externally oriented thinking' (EOT).

The Hospital Anxiety and Depression Scale (HADS; Zigmond \& Snaith, 1983) is a 14item self-report measure of depression and anxiety; seven items relate to symptoms of depression and seven to symptoms of anxiety. Each item consists of a statement, e.g. "I feel cheerful" followed by four possible responses indicating the extent of agreement with the statement. The range of scores on each item are $0-3$, thus the range of scores on each subscale is $0-21$ with higher scores indicating greater depression and anxiety. Both subscales have been shown to have good reliability (.7) and validity, as they have been shown to correlate positively (.7) with clinicians' ratings of the severity of symptoms (Zigmond \& Snaith, 1983).

The Digit Symbol Substitution Task and the Digit Span Task, both of which are from the WAIS-R IQ test (Wechsler, 1981), were used as a distraction during the 5-minute filled delay period between the encoding and recognition memory phases.

\section{Emotional faces}

Three hundred and twenty photographic images were drawn from the Karolinska (Lundqvist, Flyktam \& Ohman, 1998) and Nimstim (Tottenham et al., 2009) face sets. These images featured forty males and forty females each portraying four different emotional facial expressions (happiness, sadness, anger and neutral affect). The faces were pseudo-randomly assigned to subsets (each containing 10 happy, 10 sad, 10 angry and 10 neutral faces, with each emotion being portrayed by five different males and females). The external features (e.g. hair) was digitally removed from each face, to ensure that participants could not use variations in external facial features to make their recognition memory judgements, and then all images were transformed to greyscale. Faces differed slightly in size due to differences in the original image sizes but were all approximately 380 pixels x 400 pixels. These faces have been shown to have good face validity, with an average recognition rate for these four emotions of $88 \%$ (range 83 
to 92) for the NIMSTIM (Tottenham et al., 2009) and 79\% (range 62 to 92) for the KDEF (Goeleven et al., 2008). They are also reliable with a Kappa of .84 for the NIMSTIM. Average Test re-test matches for labelling of the KDEF faces was 91\% (ranging from 84 to 97). For both face-sets it has consistently been shown that angry, happy and sad expressions are matched for arousal levels, with neutral faces being rated as significantly less arousing (Adolph \& Alpers, 2010; Czerwon et al., 2011; Goeleven et al., 2008).

\section{Procedure}

During the encoding phase, participants were presented with a random sequence of forty faces (10 happy, $10 \mathrm{sad}, 10$ angry and 10 neutral), via a computer with a 19" monitor, and were asked to identify the emotion portrayed by each face - a forced choice from happiness, sadness, anger or neutral affect. They were also informed that they their memory for these faces would be tested later in the study (in line with DiStefano \& Koven, 2012). Superlab Pro 2.0.4 was used to present the stimuli and to record the participants' accuracy and response times (in milliseconds). Each trial began with a fixation cross (presented centrally for 500 milliseconds), followed by a face (presented centrally) and four emotional labels (presented horizontally across the screen below the face in a new random order for each trial), which remained on screen until a response was made. Participants were asked to press the key $(z, v, m$, or $/)$ that corresponded to the location of the label that matched the emotion shown by the face. Once all faces had been presented there was a five-minute delay, during which time the participants were asked to complete the Digit Symbol Substitution Task and Digit Span Task. Participants were subsequently given an intentional recognition memory test for the faces. During this task, participants were presented with the forty faces they had previously viewed intermingled with 
forty 'foils' - consisting of 10 happy, 10 sad, 10 angry and 10 neutral faces ${ }^{3}$ - and were asked to make old/new and remember/ know/ guess decisions for each face. Each trial began with a centrally presented fixation cross (for $500 \mathrm{~ms}$ ), followed by a face which remained in the centre of the screen until a response was made. Participants pressed ' $z$ ' for 'old' faces they recognised from the encoding phase and ' $v$ ' for 'new' faces that they did not recognise. They were also asked to make 'Remember', 'Know' or 'Guess' judgements about any faces that they recognised. Remember was defined to the participants as 'having a specific memory of the time the face appeared on the screen, such as a particular image or thought associated with the face, or the feeling state experienced when viewing the face'. A Know response was defined as 'knowing the face was part of the original set but not being able to retrieve more memories' and a Guess response as 'a situation where you think it was plausible that the face was presented in the original set but you do not have any degree of certainty'. Finally, participants were asked to complete the HADS and TAS-20.

\section{Data Analysis}

The percentage of correctly recognised emotional expressions and the mean emotion recognition times (in milliseconds) were calculated for each type of emotional expression. Recognition memory was examined using signal detection analysis, with proportion of correctly recognised faces 'hits' and proportion of incorrectly recognised 'novel' faces (false positives) used to calculate measures of memory sensitivity (d') and response bias (C) for emotional and neutral faces. The advantage of using signal detection analysis in recognition memory is that it provides a measure (d') of 'pure' memory sensitivity that is uncontaminated by the participants' response bias (Parks, 1966). In addition, the number of Remember, Know

\footnotetext{
${ }^{3}$ The faces presented at encoding and as 'foils' during the recognition memory test were counterbalanced to ensure that each individual poser appeared equally often as a 'target' and 'foil' and equally often with a sad, happy, angry or neutral expression.
} 
and Guess responses were recorded for emotional and neutral faces. Differences in these metrics between emotional and neutral faces were assessed using a series of paired samples ttests (or Wilcoxon tests where parametric assumptions were not met). The influence of alexithymia, controlling for age and mood, on emotion recognition and recognition memory performance was established using a series of hierarchical multiple regressions with age and mood (depression and anxiety) at Step 1 and alexithymia (the three subscales of the TAS-20) at Step 2.

\section{Results}

\section{Participant Characteristics}

Mean age of the participants in Study 1 and their alexithymia, depression and anxiety scores are presented in Table 1.

\section{Facial Emotion Recognition}

Analysis of emotion recognition accuracy revealed a significant main effect of emotion, $\mathrm{F}(2.3,86.5)=7.87, \mathrm{p}<.001 ; \mathrm{\eta}^{2} \mathrm{p}=.17$ (Greenhouse Geisser), with happy expressions being recognised more accurately $(\mathrm{Mean}=97.43, \mathrm{SD}=5.5)$ than angry $(\mathrm{M}=90, \mathrm{SD}=10)$, neutral $(\mathrm{M}=86.15, \mathrm{SD}=16.3)$ and sad faces $(\mathrm{M}=88.21, \mathrm{SD}=10.5)$, all tests $\mathrm{p}<.001$ (Bonferroni corrected). Analysis of reactions times for emotion recognition judgements revealed a significant main effect of emotion; $\mathrm{F}(1.7,63.7)=3.41, \mathrm{p}<.05 ; \eta^{2} \mathrm{p}=.08$ (Greenhouse Geisser), with happy expressions being recognised more quickly $(\mathrm{M}=2206.18 \mathrm{~ms}, \mathrm{SD}=562)$ than angry $(\mathrm{M}=2569.13 \mathrm{~ms}, \mathrm{SD}=674)$, neutral $(\mathrm{M}=2499.31 \mathrm{~ms}, \mathrm{SD}=658)$ and sad faces $(\mathrm{M}=2523 \mathrm{~ms}$, $\mathrm{SD}=1059)$, all tests $\mathrm{p}<.01$ (Bonferroni corrected).

After controlling for age and mood, none of the facets of alexithymia predicted emotion recognition accuracy or latencies, all tests $p>.05$. However, these analyses did reveal that 
depression significantly predicted emotion recognition latencies for angry $(\beta=.47, p=.016)$, happy $(\beta=.54, p=.002), \operatorname{Sad}(\beta=.54, p=.001)$ and neutral expressions $(\beta=.64, p=.001)$.

\section{Memory for Emotional Faces ${ }^{4}$}

Analysis of the recognition memory metrics (see Table 2) revealed no significant differences between emotional and neutral faces on all memory scores, all tests $\mathrm{p}>.05$.

The results of a hierarchical regression revealed that age and mood combined explained around about $12 \%$ of the variance in d' scores for emotional faces (see Table 3 ); $\mathrm{R}^{2}=.12, \mathrm{~F}(1$, $36)=1.53, p=.22$. At Step 2, alexithymia explained an additional $32 \%$ of the variance in d' scores for emotional faces, which was significant; $\mathrm{R}^{2} \Delta=.32, \mathrm{~F}(3,31)=5.93, \mathrm{p}=.003$. DIF and DDF scores both entered as significant predictors; such that higher DIF scores were associated with better memory for emotional faces (d'scores); $\beta=.60, p=.001$ and higher DDF scores were associated with poorer memory (d') for emotional faces; $\beta=-.53, p=.009$. Further analysis using partial correlations (controlling for age and mood) revealed that d' values for angry faces were negatively associated with DDF scores; $r(33)=-.44, p=.008$ and d' values for sad faces were positively related to DIF scores; $r(33)=.25, p=.07$. There were no other significant relationships, all tests $\mathrm{p}>.3$. Analysis of the number of recognition memory hits for emotional faces (see Table 3) revealed that age and mood explained a negligible amount $(<1 \%)$ of the variance in the number of hits for emotional faces, $\mathrm{F}<1$. At step 2, alexithymia explained an additional $13 \%$ of the variance in the number of hits; $\mathrm{R}^{2} \Delta=.32, \mathrm{~F}(3$, $31)=1.5, \mathrm{p}=.21$. Follow up analyses using partial correlations, controlling for age and mood, revealed that DDF scores were negatively associated with the number of hits for angry faces; $r(33)=-.39, p=.02$, but DDF scores were not significantly related to the number of hits for

\footnotetext{
${ }^{4}$ Memory for faces was not related to facial emotion recognition performance ( $\%$ correct or response times) at encoding, all tests $\mathrm{p}>.05$.
} 
happy, sad of neutral faces, all tests $p>.05$. After controlling for age and mood, none of the facets of alexithymia significantly predicted d', remember, know, or guess scores for neutral faces, and know or guess scores for emotional faces, all tests $\mathrm{p}>.05$. However, after controlling for mood, there was a non-significant trend towards a negative relationship between DDF and remember scores for emotional faces, $r(35)=-.26, p=.057$. Follow up analyses confirmed that remember scores for angry faces were negatively related to DDF scores, $\mathrm{r}(36)=-.24, \mathrm{p}=.07$, but remember scores for happy, sad or neutral faces were not related to DDF scores, all tests $\mathrm{p}>.05$.

\section{Discussion}

\section{Facial emotion recognition}

It was predicted that emotion recognition accuracy would be negatively associated with scores on the TAS-20 and emotion recognition latencies would be positively related to alexithymia. These predictions were not supported by our data, as neither emotion recognition accuracy nor latencies were related to alexithymia. These findings are inconsistent with previous evidence showing impaired facial emotion recognition in alexithymia (Grynberg et al., 2012). However, our results are congruent with findings from several clinical and healthy samples showing no emotion recognition deficits in participants with elevated alexithymia scores (Kessler et al., 2006; Prkachin, Casey, \& Prkachin, 2009; Ridout et al., 2012; Sharpe et al., 2016; Wallis et al., 2018). The variation in findings might be a consequence of differences in the emotion recognition tasks used in the respective studies. For example, Parker, Prkachin and Prkachin (2005) reported that emotion recognition deficits in alexithymia were more evident when participants were put under time pressure (i.e. by presenting the faces for only a short duration). Furthermore, Kätsyri et al (2008) demonstrated that high alexithymia scorers exhibited emotion recognition deficits 
when faces were spatially degraded, but not when non-degraded images were used. Starita et al. (2018) reported that emotion recognition deficits in alexithymia were more evident for low intensity emotional expressions. With this in mind, it would seem likely that the current task may not have been sufficiently challenging to result in emotion recognition deficits in those with higher alexithymia scores. It should also be noted that the use of a forced choice paradigm may not be ideal for revealing of the kind of emotion recognition difficulties that occur in everyday social situations (Russell, 1993).

Memory for faces

It was expected that memory for faces would be negatively related to alexithymia. However, this relationship was only expected for emotional and not neutral faces. The current findings for neutral faces were congruent with these predictions, as none of the memory metrics for neutral faces were associated with alexithymia. This is consistent with previous studies using words (Luminet et al., 2006; Suslow et al., 2003; Vermeulen \& Luminet, 2009) and neutral faces (Di Stefano \& Koven, 2012), but differs from Correro et al (2019), who reported evidence of impaired memory for neutral words and stories in high alexithymia scorers. Memory performance for emotional faces was also consistent with our predictions, as d' scores and the number of hits for emotional faces were negatively related to scores on the DDF subscale of the TAS-20. Interestingly, follow-up analyses revealed that these relationships were limited to angry faces and not sad or happy. Importantly, DDF scores were associated with lower Remember scores for angry faces, which suggests that alexithymia is linked to impaired conscious recollection of the angry faces. The current findings suggest that the memory deficit for affective words reported in previous studies (Luminet et al., 2006; Suslow et al., 2003; Vermeulen \& Luminet, 2009) generalises to the processing of emotional faces in alexithymia. However, it would appear that there is some variability in the facet(s) of alexithymia that is (are) associated with memory deficits for emotional material. In some 
previous studies (Suslow et al., 2003; Vermeulen \& Luminet, 2009) memory for emotional words was negatively associated with DIF scores, whereas in Luminet et al. (2006) memory for emotional words was negatively related to total TAS-20 scores, and in the current study, memory for emotional faces was negatively related to DDF scores. The current findings also represent an important development of the recent work examining memory for non-verbal stimuli in alexithymia (Di Stefano \& Koven, 2012; Takahashi et al., 2015). Di Stefano and Koven (2012) reported that high alexithymia scorers exhibited impaired memory for people in social scenes, but as the emotional expressions featured in these images were not varied systematically it was not possible to ascertain if alexithymia impaired memory for specific emotional expressions. The current findings extend this work by confirming that alexithymia specifically impairs conscious memory for angry faces. There would also appear to be a difference in the pattern of deficits exhibited by high alexithymia scorers in short- and longterm memory. In the current study, deficits in long-term memory were most evident for angry faces, whereas participants with elevated alexithymia scores exhibited impaired short-term memory for happy faces (Takahashi et al., 2015). However, this might also relate to differences in the facial stimuli used in the two studies; Takahashi and colleagues used schematic representations of facial expressions, whereas we used validated photographs of facial expressions.

As it has been proposed that individuals with high levels of alexithymia have less elaborated/ integrated emotional schema (Luminet et al., 2006; Suslow et al., 2003), it is plausible that angry faces might have been perceived as less conceptually salient by participants with high alexithymia (DDF) scores. This is a particularly relevant for the findings relating to Remember scores, as conceptual salience has been identified as a critical factor in the remembering experience (Rajaram, 1998). However, if emotional faces were less salient to individuals with high alexithymia scores then we would have expected to see 
differences in the processing of these stimuli during encoding, yet these differences were not evident in the emotion recognition latencies or accuracy scores. Nevertheless, it is plausible that alexithymia could have influenced processing of the faces at encoding in ways that did not influence response times and performance; for example, individuals with high DDF scores might have spent less time looking at the eyes than did low scorers. In line with this suggestion, Fujiwara (2018) used an eye tracker and demonstrated that individuals with high levels of alexithymia tended to avoid looking at eyes, which led the author to conclude that eye contact might be emotionally challenging for individuals with high alexithymia - hence they avoid looking at eyes. This is notable, as reduced eye movements when viewing faces has been shown to influence memory for angry faces (Wells, Beevers, Robison, \& Ellis, 2010).

In contrast to studies showing a negative relationship between DIF scores and memory for affective words (e.g. Suslow et al, 2003; Vermeulen \& Luminet, 2009), current findings revealed that DIF scores were positively related to memory for sad faces. This finding might also be a consequence of alexithymia-related differences in the perceived salience of the faces. For example, the sad faces might have been considered as more salient by those with high DIF scores. In line with this proposal, Metzler and Nielson (2010) reported that whilst high alexithymia scorers demonstrated impaired memory for emotional words, they actually exhibited a memory advantage for words connoting illness, which the authors concluded was due to differences in the relative salience of the material.

\section{Methodological considerations}

It is notable that participants in the current study were informed that their memory was going to be tested for the faces (in line with DiStefano \& Koven, 2012), whereas studies examining memory for emotional and neutral words have tended to use an incidental learning paradigm, 
where participants were not prior warned about the memory test. This is likely to have influenced how the participants processed the faces at encoding, as in addition to making emotion recognition judgements they might also have been looking for distinctive elements of each face that could aid their memory at later testing. It is also notable that the delay (of five minutes) between encoding and retrieval was relatively short in comparison to some previous studies (e.g. DiStefano \& Koven (2012) who used 45 minutes, and Nielson \& Meltzer (2009) who used 24 hours). Nevertheless, the current delay period is consistent with other studies (e.g. Luminet et al., 2006; Ridout et al., 2003; Ridout et al., 2009ab). A further limitation of the study was that we did not pilot test the faces for valence and arousal. Neither did we get participants to rate their level of arousal in response to the stimuli. However, several studies using the NIMSTIM and KDEF face sets have demonstrated that angry, sad, and happy faces differ in valence ratings but not in arousal. Moreover, all three emotions were rated as more arousing that neutral faces (Adolph \& Alpers, 2010; Czerwon et al., 2011; Goeleven et al., 2008).

In sum, the findings of Study 1 revealed that alexithymia (DDF) scores were negatively associated with memory (hits and d' scores) for emotional, but not neutral faces, particularly when the faces expressed anger. This pattern was evident for Remember but not Know scores, which suggests that alexithymia impaired conscious recollection of angry faces. It is plausible that the angry faces may have been less conceptually salient for the high alexithymia scorers in comparison to the low scorers, which would have reduced the distinctiveness of these faces at encoding. On the other hand, sad faces may have been perceived as more salient, which might account for the finding of a positive relationship between DIF scores and memory for sad faces. The current findings are important, as they extend previous work examining memory for non-verbal material in alexithymia and demonstrate that the memory deficit observed for affective words generalises to the processing of important non-verbal socially 
relevant stimuli (i.e. emotional faces). As noted by Takahashi et al. (2015) biased memory for faces in alexithymia might impair normal social functioning, which in turn might undermine key social bonds. This is important, as social support has shown to be vital in protecting against psychological distress.

\section{Study 2}

The findings of Study 1 demonstrated that DDF scores were negatively associated with memory (d', hits, and remember scores) for emotional, but not neutral faces. This suggests that the memory deficit shown in high alexithymia scorers for affective words generalises to non-verbal socially relevant stimuli. This is the first study to directly test this, although the findings of DiStefano and Koven (2012) could also be interpreted as evidence of impaired memory for emotional but not neutral non-verbal stimuli. In their study, individuals with high levels of alexithymia exhibited impaired memory for images of people in social scenarios (which included some emotional content), but intact memory for neutral faces. This is important, as this memory deficit for important social stimuli could have implications for social functioning of individuals with high levels of alexithymia (Takahashi et al., 2015). However, there were several limitations to their study. Firstly, the two types of stimuli varied on factors other than emotional content (e.g. the social images were more complex), which makes it difficult to draw firm conclusions from their findings. Furthermore, they did not systematically manipulate the emotional content of the social interactions. Finally, in line with the faces from Study 1 their stimuli were static, whereas in everyday interactions the social signals are dynamic and rapidly changing. With this in mind, it is important to determine if alexithymia influences memory for dynamic social interactions.

The Awareness of Social Inference Test (TASIT; McDonald et al., 2003) features short video clips of individuals in realistic social interactions and includes protagonists displaying different emotions, as well as neutral affect. It is also notable, that McDonald et al 
(2006) reported that performance on this task in healthy participants was positively related to memory for faces (.69) and to measures of facial emotion processing (Ekman faces task) both recognition (.69) and matching (.7). Thus, these stimuli would seem to be ideal for the purposes of the proposed study. Given that previous work from our group has shown that alexithymia was negatively associated with the recognition of emotion from these realistic social displays (Ridout, Thom \& Wallis, 2010) and given the findings of Study 1 regarding static faces, it is plausible that this alexithymia might also influence memory for these complex social stimuli.

Overview and predictions

The aim of Study 2 was to determine if variations in alexithymia were associated with differences in memory for videos of realistic social interactions. To address this question a different sample of female undergraduates was presented with a series of short video clips of social interactions and were asked to identify the emotion portrayed by the central (or lone) protagonist in each video clip (forced choice from angry, happy, neutral or sad). After a 5minute filled delay, participants were given an intentional recognition memory test for the video clips they had viewed at encoding. Participants also completed the TAS-20 and HADS questionnaires.

In line with Ridout et al. (2010), it was expected that the percentage of correctly recognised emotional displays would be negatively related to alexithymia. In line with previous findings using non-verbal stimuli (Di Stefano \& Koven, 2012) and the results of Study 1 , it was expected that memory for emotional, but not neutral video clips would be negatively related to alexithymia. The relationship was expected to be more evident in the Remember than Know responses. Note: these findings were expected to be independent of the influence of age and mood (depression and anxiety). 


\section{Method}

Participants

Thirty-eight ${ }^{5}$ female undergraduates took part in exchange for course credit ${ }^{6}$. The mean age of the sample was 19.63 years $(\mathrm{SD}=2.7)$. Individuals with a history of psychiatric illness were requested not to take part in study. Participants provided full-written informed consent before taking part. The study was approved by the Psychology Subcommittee of Aston University's Research Ethics Committee.

Measures and tasks

Alexithymia was measured using the 20-item Toronto Alexithymia Scale (TAS-20; Bagby et al., 1994) and mood was assessed using the Hospital Anxiety and Depression Scale (HADS; Zigmond \& Snaith, 1983). As in study 1, the Digit Symbol Substitution Task and the Digit Span Task from the WAIS-R (Wechsler, 1981) were included as distractor tasks during the filled delay between encoding and recognition memory testing.

Video clips

Thirty-two video clips were drawn from the Emotion Evaluation phase of The Awareness of Social Inference Test (TASIT; McDonald et al., 2003). Each clip featured either a social interaction between two protagonists or a single speaker - on the phone or directly addressing the viewer. During each clip the central (or lone) protagonist expresses one of four emotions (sadness, happiness, anger or neutral affect). Each emotion was featured

\footnotetext{
${ }^{5}$ Based on the observed effect sizes reported in Study 1 and in a previous study examining the influence of alexithymia on memory for faces (DiStefano \& Koven, 2012) a large effect size $\left(\mathrm{f}^{2}=.35\right)$ was expected. The results of a power calculation using $\mathrm{G}^{*}$ Power to determine the required sample size to detect a significant change in $\mathrm{R}^{2}$ on a multiple regression with three tested predictors and a total of six predictors revealed that a minimum of 36 participants would be required to detect a large effect size with a power of .8 and an alpha level of .05. Therefore, this study was sufficiently powered.

${ }^{6}$ Two male participants were also recruited. However, given that males and females differ in face processing (Sullivan et al., 2015) and alexithymia (Levant et al., 2009), and that there were an insufficient number of males to allow for a reliable group comparison we chose to exclude these participants from the analysis of the data from Study2.
} 
in eight video clips, four featuring a male protagonist and four featuring a female. The social interactions were performed by professional actors and the average percentage agreement, obtained from a large community sample ( $\mathrm{n}>250)$ was $82 \%$ for the different emotions. Furthermore, McDonald et al (2006) reported that emotion recognition from these videos correlated positively with performance on the Ekman task, both emotion matching (.7) and emotion recognition (.69) - all of which suggests that the emotions portrayed in the videos are valid. Given that video clips in TASIT varied in length, each clip was edited to 15 seconds (containing the most relevant emotional components) in order to reduce inter stimulus differences, which may have influenced memory performance. Twenty clips (five each of the neutral, sad, angry \& happy videos), ten featuring a male and ten featuring a female protagonist were pseudo-randomly selected to be presented during the encoding stage. The remaining 12 clips (three each of the neutral, sad, angry \& happy videos) were used as novel 'foils' during the recognition memory task ${ }^{7}$.

\section{Procedure}

During the encoding phase, participants were shown a PowerPoint presentation featuring the twenty video clips in a fixed randomised order and were asked to identify the emotion portrayed by the central (or lone) protagonist in each clip, by choosing the most appropriate emotion label from a choice of four (happiness, sadness, anger or neutral affect), which were presented in a random order for each trial. They were given unlimited time to select the appropriate emotion and were able to control the onset of the next clip. The label chosen for each trial was recorded by the experimenter using a modified version of the TASIT scoresheet. Participants were also informed that their memory for the video clips

\footnotetext{
7 The video clips presented at encoding and as 'foils' during the recognition memory test were counterbalanced across participants to ensure that, as far as possible, each individual actor appeared equally often as a 'target' and 'foil' and equally often when expressing sadness, happiness, anger or neutral affect.
} 
would be tested later in the study. Once all clips had been presented, there was a 5-minute filled delay during which time the participants were invited to complete the Digit Symbol Substitution Task and the Digit Span Task. Participants were subsequently given an intentional recognition memory test for the video clips they had viewed at encoding, during which they were shown a PowerPoint presentation featuring the twenty clips they had previously viewed intermingled with twelve novel 'foils' and were asked to make an old/new judgement for each clip. For every clip that the participant recognised they were asked to make remember/ know/ guess judgements. Finally, they were invited to complete the TAS-20 and the HADS.

\section{Data analysis}

The percentage of correctly identified emotional displays was calculated for each type of social interaction (angry, happy, neutral and sad), which were analysed using a one-way repeated measures ANOVA, with emotion entered as the within subjects' factor. Pairwise comparisons were conducted using Bonferroni tests (to adjust alpha for multiple comparisons). The mean number of correctly recognised video clips (hits) ${ }^{8}$, the number of falsely recognised clips (false positives), and the number of Remember and Know responses for emotional and neutral clips were compared using paired t-tests (or Wilcoxon tests where parametric assumptions were not met). Hierarchical multiple regression, with age entered at Step 1, mood (depression and anxiety) entered at Step 2 and alexithymia (scores on the three subscales of the TAS-20) entered at Step 3, was used to determine the influence of alexithymia on emotion recognition and recognition memory performance.

\footnotetext{
${ }^{8}$ We had planned to conduct signal detection analysis on the data from Study 2, but initial inspection of the data revealed that hits were close to ceiling and false positives close to floor, thus signal detection analysis would have been unreliable. Therefore, we analysed the hits and false positives separately. Furthermore, the number of guess responses was extremely low, therefore these data are not reported or analysed.
} 


\section{Results}

Participant Characteristics

Mean age of the participants in Study 2 and their alexithymia, depression and anxiety scores are presented in Table 4.

\section{Emotion recognition}

Analysis of emotion recognition scores revealed a significant main effect of type of emotion $\left(\mathrm{F}(2.4,88.8)=38.5, \mathrm{p}<.001, \mathrm{\eta}_{\mathrm{p}}^{2}=.51\right.$ (Greenhouse Geisser), with fewer neutral interactions being correctly identified $(\mathrm{M}=77.37, \mathrm{SD}=14.1)$ than happy $(\mathrm{M}=96.3, \mathrm{SD}=7.9)$, angry

$(\mathrm{M}=97.90, \mathrm{SD}=6.2)$ or $\mathrm{sad}(\mathrm{M}=95.26, \mathrm{SD}=8.6)$, all tests $\mathrm{p}<.001$ (Bonferroni corrected).

\section{The influence of alexithymia on emotion recognition}

Hierarchical regression (see Table 5) revealed that age and mood had a negligible influence on the recognition of anger; $\mathrm{R}^{2}=.06 ; \mathrm{F}(2,34)=.66, \mathrm{p}=.58$. The addition of alexithymia at Step 2 resulted in a non-significant increase in variance explained; $\mathrm{R}^{2} \Delta=.14 ; \mathrm{F}(3,31)=1.8, \mathrm{p}=.16$. Nevertheless, DIF entered as a significant predictor of emotion recognition scores for angry videos; $\beta=-.49, p=.031$ but alexithymia did not influence the recognition of happiness, sadness or neutral affect from the video clips; all tests $\mathrm{p}>.05$.

Memory for video clips

Analysis of the recognition memory data (see Table 6) revealed that participants made a greater number of correct recognitions (hits) for neutral clips than emotional. Similarly, participants made a greater number of Remember judgements for neutral clips compared to emotional. However, participants made a greater number of false recognitions of emotional foils than neutral. Participants did not differ in the number of Know judgements they made for emotional and neutral clips. 


\section{The influence of alexithymia on memory for video clips}

Hierarchical regression (see Table 7) revealed that age and mood explained about $20 \%$ of the variance in the number of hits for emotional videos; $\mathrm{R}^{2}=.21, \mathrm{~F}(2,34)=2.96, \mathrm{p}=.04$. Depression entered as the only significant predictor, $\beta=-.49, p=.006$. The addition of alexithymia at Step 2 explained an additional $23 \%$ of the variance in the number of emotional video clips that were correctly recognised at memory testing, which was significant; $\mathrm{R}^{2} \Delta=$ $.23, \mathrm{~F}(3,31)=4.18, \mathrm{p}=.014$. At Step 2 , the influence of depression was no longer significant, $\beta=-.35, p=.08$ but DIF and EOT both entered as significant predictors of memory for emotional interactions, $\beta=-.41, p=.03$ and $\beta=-.33, p=.044$.

Hierarchical regression (see Table 7) revealed that age and mood explained $9 \%$ of the variance in the Remember scores for emotional video clips; $\mathrm{R}^{2}=.09, \mathrm{~F}(2,34)=1.1, \mathrm{p}=.38$. The addition of the alexithymia scores at step 2 explained an additional $18 \%$ of the Remember scores for emotional video clips, but this did not represent a significant increase in variance; $\mathrm{R}^{2} \Delta=.18, \mathrm{~F}(3,31)=2.54, \mathrm{p}=.075$. At Step 2, no factors entered as significant predictors, but there was a trend for a negative relationship between DIF and Remember scores for emotional clips; $\beta=-.39, p=.07$. Follow up analyses using partial correlations, controlling for age and mood, revealed that DIF scores were negatively associated with Remember scores for happy and angry videos; $r(33)=-.36, p=.032$ and $r(33)=-40, p=.019$, but not sad; $\mathrm{r}(33)=-.21, \mathrm{p}=.27$.

After controlling for age and mood, alexithymia did not significantly influence the number of hits for neutral videos, false positives for emotional or neutral clips, Remember scores for neutral videos, or Know scores for emotional scenes, all tests $\mathrm{p}>$.05. However, alexithymia did influence the number of Know responses for neutral videos (see Table 8). Age and mood explained $9 \%$ of the variance in Know scores for neutral videos; $\mathrm{R}^{2}=.09, \mathrm{~F}(2,34)=1.1$, 
$\mathrm{p}=.37$. The addition of alexithymia at Step 2 explained an additional $29 \%$ of the variance; $\mathrm{R}^{2}$ $\Delta=.29 ; \mathrm{F}(3,31)=3.0, \mathrm{p}=.043$. At Step 2 , DIF scores entered as the only significant predictor of Know scores for neutral videos; $\beta=.54, \mathrm{p}=.012$.

\section{Relationship between emotion recognition and memory performance}

The total emotion recognition score was positively related to the number of hits for emotional, but not neutral videos; $\mathrm{r}(38)=.44, \mathrm{p}=.006$ and $\mathrm{r}(38)=-.07, \mathrm{p}=.66$ respectively. Total emotion recognition score was also positively related to the Remember scores for both emotional and neutral videos; $r(38)=.34, p=.03$ and $r(38)=.36, p=.025$. Separate analyses by valence revealed that the percentage of angry videos correctly labelled at encoding was positively related to the number of Remember scores for angry videos; $r(38)=.33, p=.04$. Similarly, the number of neutral video clips that were correctly labelled was positively related to the Remember scores for neutral videos, $\mathrm{r}(38)=.35, \mathrm{p}=.033$.

\section{Discussion}

\section{Emotion recognition}

It was expected that the percentage of correctly recognised emotional displays would be negatively related to alexithymia. Our findings were only partially consistent with this expectation, as alexithymia (DIF) scores were only negatively related to the recognition of anger and not happiness, sadness or neutral affect. This differs from our previous finding that alexithymia was associated with a general deficit in the recognition of emotion from these videos (Ridout et al., 2010). One possible explanation concerns the fact that the video clips were shortened for the current study. When editing the videos we focused on the most relevant emotional elements, which might have made the emotion recognition task easier by removing some of the distracting information. In line with this argument, Ridout et al. (2007) reported that emotion recognition performance from the full-length video clips was positively 
associated with on scores on inhibitory executive tasks (Stroop and Hayling's tests). This was thought to reflect the need to inhibit the task irrelevant elements of these complex social interactions in order to make the correct emotion recognition judgements. With this in mind it is notable that facets of alexithymia have been associated with deficits in executive function, including inhibition and conflict processing (Correro et al., 2019; Koven \& Thomas, 2010; Zhang et al., 2011), thus the observed deficit in anger recognition might plausibly be a consequence of impaired executive function in those with high alexithymia.

Another possibility is that high DIF scorers may have allocated fewer attentional resources to the processing of the angry videos. In line with this argument, Vermeulen et al. (2008) reported that participants with high alexithymia scores exhibited lower activity in the attentional components of the event related potential (N2b and P3a) in response to angry faces, which the authors interpreted as evidence of abnormal attentional processing of anger in alexithymia. Furthermore, Vermeulen, Luminet, and Corneille (2006) reported reduced affective priming from angry faces in participants with high alexithymia, which suggests angry expressions were not automatically processed by these individuals. Thus, the current findings support the growing body of work showing a deficit in anger processing in participants with high levels of alexithymia.

\section{Memory for social interactions}

It was predicted that alexithymia would be negatively associated with memory for emotional, but not neutral video clips. In line with these predictions, none of the memory metrics for neutral scenarios were related to alexithymia, with the exception of a positive relationship with Know responses (see below). These findings are consistent with Study 1 and with studies examining memory for neutral faces (Di Stefano \& Koven, 2012) and words (Luminet et al., 2006; Suslow et al., 2003; Vermeulen et al., 2009). However, they differ from recent 
evidence of an alexithymia-related deficit in memory for neutral stimuli (Correro et al., 2019). The finding that higher alexithymia scores (DIF and EOT) were associated with poorer memory (hits) for emotional videos also supports our hypotheses. Furthermore, the findings that higher DIF scores were related to lower Remember scores for happy and angry videos are consistent with the prediction that the negative relationship between alexithymia and memory for emotional videos would be more evident in the Remember than Know responses. However, the lack of relationship between alexithymia and Remember scores for sad videos is inconsistent with this prediction. Taken together, our findings are congruent with previous work examining memory for words (Luminet et al., 2006; Suslow et al., 2003; Vermeulen \& Luminet, 2009). Furthermore, the current findings expand upon the work of Di Stefano and Koven (2012) and Study 1 by demonstrating that higher alexithymia scores are associated with poorer memory for dynamic social stimuli, but only when they feature emotional content.

In line with the findings of Study 1, memory deficits for the emotional video clips associated with elevated alexithymia scores might plausibly relate to differences in the perceived salience of the emotional videos. This explanation is particularly pertinent for the findings of lower Remember scores for happy and angry videos, given that conceptual salience is considered a key factor underpinning remember experiences (Rajaram, 1998). In line with this proposal, Aaron, Snodgress, Blain and Park (2018) reported that individuals with high levels of alexithymia reported lower affective responses to emotive video clips in comparison to low scorers, with high scorers often reporting 'no emotional response' to the videos. Furthermore, in the current study we observed that the ability to correctly label emotions (a conceptual processing task) during encoding was positively related to memory performance (hits and Remember scores). Specifically, the ability to correctly label angry and neutral videos was positively associated with the Remember scores for these stimuli. Thus, 
individuals with low DIF would have been able to correctly label the angry videos, presumably as their anger schema was more integrated than the high scorers, enabling them to make use of the conceptually salient aspects to encode the angry videos more distinctly. However, the finding that emotion recognition of happy videos and subsequent memory for these stimuli were not related is not consistent with this explanation. Nevertheless, evidence demonstrating reduced emotional response to appetitive words and images (Koven, 2014) suggests that lower perceived salience might still be a viable explanation for the current finding of poor memory (Remember scores) for happy videos in those with elevated DIF scores.

The current finding that DIF was positively associated with Know responses for neutral videos was not expected. Given that processing fluency is thought to underpin Know but not Remember responses (Rajaram, 1998), it would appear that individuals exhibiting elevated DIF scores were processing the neutral clips more fluently than were low scorers, perhaps due to the lack of emotional content in these stimuli.

\section{Methodological considerations}

There are a number of limitations to Study 2 that need to be considered. Firstly, the number of stimuli was quite low, which meant the initial memory set was small and the number of distractors at memory testing was even smaller. Although it is not a necessity that there are equal numbers of targets and foils, having a greater number of foils would have potentially reduced the number of hits (which was close to ceiling in the current study) and increased the number of false positives (which was close to floor in the current study). Unfortunately, this was unavoidable given the limited number of available videos from the TASIT. Future work using a larger set of videos and with an increased delay between encoding and retrieval would be expected to produce stronger findings. Another limitation is that we did not pilot 
test the images for valence and arousal ratings. In terms of valence, the reported participant agreement from a large community sample ( $\mathrm{n}>250$ ) was $>82 \%$, which suggests that the emotions portrayed were valid (McDonald et al., 2003). Furthermore, performance of a group of participants on emotion recognition from the videos correlated highly (.7) with Ekman test of emotion recognition (McDonald et al, 2006). Nevertheless, it would have been useful to ensure the emotional videos were matched for arousal and these videos were more arousing than the neutral clips. Future work examining memory for emotional videos should confirm the valence and arousal ratings to enable these factors to be included in the analysis.

In sum, the findings of Study 2 revealed that alexithymia (DIF scores) was associated with a deficit in the recognition of anger from videos of social interactions. Furthermore, memory (number of hits) for the emotional, but not neutral videos was negatively related to alexithymia (DIF and EOT) and DIF scores were negatively linked to Remember scores for angry and happy videos, which suggests that alexithymia is linked to impaired conscious recollection of these emotional videos. This represents an important extension to the previous work on memory for important socially relevant stimuli in alexithymia (e.g. DiStefano \& Koven; 2012; Takahashi et al., 2015). In line with Takahashi et al. (2015) it is plausible that deficits in the processing of these important social stimuli could have a negative impact on the social functioning of individuals with elevated alexithymia scores.

\section{General Discussion}

We conducted two experimental studies to determine if alexithymia influenced memory for emotional faces (Study 1) and realistic social interactions (Study 2). Our key findings were that, in both studies, facets of alexithymia were associated with impaired recognition memory for emotional but not neutral stimuli. Furthermore, in both studies, participants with elevated alexithymia exhibited impaired conscious recollection of the emotional stimuli, particularly 
when they featured anger. Taken together these findings are consistent with studies examining memory deficits for verbal material in alexithymia (Luminet al, 2006; Suslow et al., 2003; Vermeulen \& Luminet, 2009). A plausible explanation for these memory deficits is that individuals with elevated alexithymia might not have perceived the emotional stimuli as more conceptually salient than the neutral material. Thus, for these individuals the emotional material would not have been considered as more distinctive than the neutral stimuli. This is particularly relevant when considering the negative relationship between alexithymia and Remember responses for the emotional stimuli. This explanation is also consistent with studies examining memory deficits for emotional words in alexithymia (Luminet et al., 2006; Vermeulen et al., 2009). However, there were a number of inconsistencies in the findings of our two studies that need to be considered. Notably, whilst memory for emotional faces (Study 1) was negatively related to DDF scores, memory for emotional videos (Study 2) was negatively related to DIF and EOT. Furthermore, DIF scores were positively related to memory for sad faces (Study 1) but negatively related to memory for emotional videos (Study 2).

As noted in the discussion of Study 1, the finding of a positive relationship between DIF scores and memory for sad faces could also be explained by salience, as previous studies have demonstrated participants with elevated alexithymia can show enhanced memory for emotional material, when these stimuli were personally relevant (Melzer \& Nielson, 2010). With this in mind, it is possible that the type of stimuli used in two studies might account for the different pattern of memory in participants with high DIF scores. The emotional expressions portrayed by the faces (Study 1) were directed towards the participant, whereas in the majority of the video clips (Study 2) the affect was directed at another protagonist either seen or unseen (e.g. on the other end of a phone). Thus, it is conceivable that when the sad expressions were directed at the participants those with high DIF scores may have 
perceived them as more personally relevant than when sadness was directed at another person. Salience can also account for the influence of EOT on memory for emotional videos, as a recent study reported that this factor was associated with 'no emotional response' to affective video clips (Aaron et al., 2018). However, salience cannot explain the lack of a negative relationship between DIF scores and memory for happy and angry faces, as would have been expected from the studies examining memory for verbal material (Suslow et al., 2003, Vermeulen et al., 2009) and as was observed for happy and angry videos in Study 2.

Differences in the stimuli used in the two studies might also account for why DDF scores were negatively associated with memory for emotional faces (Study 1), but not video clips (Study 2). Fujiwara et al. (2018) reported that high alexithymia scorers avoid looking at the eye regions of angry faces. The authors proposed that direct eye contact is emotionally challenging for those with high alexithymia. Therefore, as the faces (Study 1) featured direct gaze towards the participants, they would arguably have been more emotionally challenging for the high alexithymia scorers than would the videos (Study 2), where gaze was averted. Furthermore, faces featuring threat (e.g. angry expressions) would have been expected to be particularly challenging for high alexithymia scorers. For example, it has been proposed that appraising threatening faces (fear and anger) depletes attentional resources in those with high alexithymia, due to these individuals allocating resources to regulate the negative affective impact of these stimuli (Grynberg, Vermeulen, \& Luminet, 2014; Pouga, Berthoz, de Gelder, \& Grezes, 2010). In line with this notion, Vermeulen et al. (2008) reported that higher levels of alexithymia were associated with reduced attentional processing of angry faces, indexed by lower activity in the N2b/P3a components of the EEG signal. Taken together these findings suggest that participants with high alexithymia scores would have had fewer available resources to encode the faces, which would explain the poorer memory for angry faces. However, this does not explain why it was DDF and not DIF or EOT that was related 
to poorer memory for angry faces. Although the studies demonstrating biased attentional processing of angry faces have tended to use the total TAS-20 scores rather than the subscales, there is evidence that DDF is associated with impaired processing of negative faces (Grynberg et al, 2014; Parker, Prkachin \& Prkachin; 2005). Thus, it is plausible that the previous findings relating to reduced attentional processing of anger faces could have been due to DDF rather than the other facets of alexithymia. This would explain the current findings, as DDF would have been linked to reduced attentional processing of the faces, and subsequent poorer recognition memory of these stimuli but this factor would not have been expected to influence the processing of the videos, as the gaze was averted. This could be tested using eye tracking technology to examine if there variations in viewing of faces and videos linked to facets of alexithymia and whether the pattern of eye movements predicted later memory for the stimuli. This also highlights the importance of considering the individual facets of alexithymia rather than, or in addition to, the total score.

As noted above, the negative relationship between DIF scores and memory for emotional videos, particularly those featuring anger, could feasibly be a consequence of reduced perceived salience of these stimuli for those with high DIF scores. However, another factor that might explain this finding is executive function. Importantly, this factor might also account for the variation in the findings of the two studies. Although the videos used in Study 2 were shortened and simplified, they were still more complex than the static faces used in Study 1. For example, they often involved more than one speaker, or required participants to infer the other side of a conversation (e.g. when the protagonist is speaking on the phone) and contained more task irrelevant content. Thus, appraising the videos would have made greater demands on the executive functions than would the faces. For example, Ridout et al (2007) demonstrated that emotion recognition from these videos was related to performance on measures of inhibitory function. This is notable, as recent evidence (Correro et al., 2019) 
confirmed that DIF, and not DDF, scores were negatively associated with scores on an executive factor (extracted from a battery of executive tasks). Therefore, assuming that individuals with high DIF scores also exhibit impaired executive function, this would explain the observed negative relationship between DIF scores and emotion recognition from the videos (in line with Ridout et al., 2007) and the negative association between DIF scores and memory for the videos, as these individuals would have had fewer resources to allocate to the encoding of the videos.

\section{Alternative explanations and other considerations}

Another factor that might account for the differential effects of alexithymia on memory for emotional and neutral material is arousal. Arousal is proposed to play a key role in the longterm consolidation of emotional memories (Sharot, Delgado \& Phelps, 2004). This factor is also thought to exert an influence at the time of memory encoding by modifying cognitive processes, such as attention (Talmi, Schimmack, Paterson, \& Moscovitch, 2007), which is thought to explain the advantage for emotional material over neutral at immediate memory testing. With this in mind, it is notable that there is evidence of reduced physiological arousal in alexithymia from studies using skin conductance (e.g. Constantinou et al., 2013; Franz et al., 2003), heart rate (e.g. Franz et al., 2003) and facial electromyography (EMG) (e.g. Denise, 2009; Scarpazza, 2015). However, this also some evidence of hyperarousal (e.g. Davydov et al., 2013; Luminet et al, 2004) and other studies showing no arousal differences between high and low alexithymia scorers (e.g. Gilbert, 2008; Stone \& Nielson, 2001). In a recent review, Panayiotou, Panteli and Vlemincx (2018) concluded that, although there are several contradictory findings, the weight of evidence supports there being no difference in physiological arousal between high and low alexithymia scorers. Nevertheless, they concluded that hypo-arousal was often found when tasks were emotional in nature (e.g. appraising images or films). With this mind, future work could examine the role that arousal plays in these 
alexithymia-related memory deficits using physiological measurements of arousal such as galvanic skin response, heart rate or facial EMG.

In summary, the findings of two experimental studies demonstrate that elevated levels of alexithymia are associated with poorer memory for emotional non-verbal socially relevant stimuli, whereas memory for neutral stimuli was unaffected. These deficits were particularly evident in the conscious recollection of emotional stimuli, especially those featuring anger. However, the findings of the two studies were related to different facets of alexithymia, as high DDF scores were associated with impaired memory for emotional faces (Study 1) and high DIF scores were linked to lower memory for emotional videos (Study 2). These differences are thought to be related to the nature of the emotional stimuli. Finally, in line with Takahashi et al (2015), it is proposed that impaired memory for these important social stimuli could have a negative impact on the social functioning of individuals with elevated alexithymia. 


\section{References}

Aaron, R. V., Snodgress, M. A., Blain, S. D., \& Park, S. (2018). Affect labeling and other aspects of emotional experiences in relation to alexithymia following standardized emotion inductions. Psychiatry Research, 262, 115-123.

Adolph, D., \& Alpers, G. W. (2010). Valence and arousal: a comparison of two sets of emotional facial expressions. American Journal of Psychology, 123(2), 209-219.

Bagby, R. M., Parker, J. D. A., \& Taylor, G. J. (1994a). The 20-Item Toronto-AlexithymiaScale. 1. Item selection and cross-validation of the factor structure. Journal of Psychosomatic Research, 38(2), 3-32.

Bagby, R. M., Taylor, G. J., \& Parker, J. D. A. (1994b). The 20-Item Toronto-AlexithymiaScale. 2. Convergent, discriminant, and concurrent validity. Journal of Psychosomatic Research, 38(3), 3-40.

Constantinou, E., Panayiotou, G., \& Theodorou, M. (2014). Emotion processing deficits in alexithymia and response to a depth of processing intervention. Biological psychology, 103, 212-222.

Correro II, A. N., Paitel, E. R., Byers, S. J. \& Nielson, K. A. (2019): The role of alexithymia in memory and executive functioning across the lifespan, Cognition and Emotion, DOI: $10.1080 / 02699931.2019 .1659232$

Czerwon, B., Lüttke, S., \& Werheid, K. (2011). Age differences in valence judgments of emotional faces: The influence of personality traits and current mood. Experimental Aging Research, 37(5), 503-515.

Davydov, D. M., Luminet, O., \& Zech, E. (2013). An externally oriented style of thinking as a moderator of responses to affective films in women. International Journal of Psychophysiology, 87(2), 152-164.

DiStefano, R. A. \& Koven, N. S (2012). Dysfunctional emotion processing may explain visual memory deficits in alexithymia. Personality and Individual Differences, 52, 611-615.

Franz, M., Schaefer, R., \& Schneider, C. (2003). Psychophysiological response patterns of high and low alexithymics under mental and emotional load conditions. Journal of Psychophysiology, 17(4), 203-213.

Fujiwara, E. (2018). Looking at the eyes interferes with facial emotion recognition in alexithymia. Journal of Abnormal Psychology, 127(6), 571.

Gardiner, J. M. (1988). Functional aspects of recollective experience. Memory \& Cognition, 16, 309-313.

Gilbert, A. M. (2008). The physiological response to implicit and explicit fear faces in alexithymia (Doctoral dissertation, University of Pittsburgh).

Goeleven, E., De Raedt, R., Leyman, L., \& Verschuere, B. (2008). The Karolinska directed emotional faces: a validation study. Cognition and emotion, 22(6), 1094-1118. 
Grynberg, D., Chang, B., Corneille, O., Maurage, P., Vermeulen, N., \& Luminet, O. (2012) Alexithymia and the Processing of Emotional Facial Expressions (EFEs): Systematic Review, Unanswered Questions and Further Perspectives. PLoS ONE 7(8): e42429.

doi:10.1371/journal.pone.0042429

Grynberg, D., Vermeulen, N. \& Luminet, O. (2014). Amplification of attentional blink by distress related facial expressions: relationships with alexithymia and affectivity.

International Journal of Psychology, 49 (5), 371-380 (2014)

Honkalampi K, Hintikka J, Tanskanen A, et al. (2000). Depression is strongly associated with alexithymia in the general population. Journal of Psychosomatic Research, 48, 99-104.

Jacob, S., \& Hautekeete, M. (1998). Alexithymia and memory: A more rigorous criterion for acceptance in recognition tasks? Encephale-Revue De Psychiatrie Clinique Biologique Et Therapeutique, 24, 199-204.

John, O. P., \& Gross, J. J. (2004). Healthy and unhealthy emotion regulation: Personality processes, individual differences, and life span development. Journal of personality, 72(6), 1301-1334.

Kätsyri, J., Saalasti, S., Tiippana, K., von Wendt, L., \& Sams, M. (2008). Impaired recognition of facial emotions from low-spatial frequencies in Asperger syndrome. Neuropsychologia, 46(7), 1888-1897.

Kessler, H., Schwarze, M., Filipic, S., Traue, H. C., \& von Wietersheim, J. (2006). Alexithymia and facial emotion recognition in patients with eating disorders. International Journal of Eating Disorders, 39(3), 245-251.

Koven, N. S. (2014). Abnormal valence differentiation in alexithymia. Personality and individual differences, 68, 102-106.

Koven, N. S., \& Thomas, W. (2010). Mapping facets of alexithymia to executive dysfunction in daily life. Personality and Individual Differences, 49(1), 24-28.

LaBar, K. S \& Cabeza, R. (2006). Cognitive neuroscience of emotional memory. Nature Reviews Neuroscience, 7(1), 54-64.

Levant, R. F., Hall, R. J., Williams, C. M., \& Hasan, N. T. (2009). Gender differences in alexithymia. Psychology of men \& masculinity, 10(3), 190.

Luminet, O., Rimé, B., Bagby, R. M., \& Taylor, G. (2004). A multimodal investigation of emotional responding in alexithymia. Cognition and emotion, 18(6), 741-766.

Luminet, O., Vermeulen, N., Demaret, C., Bagby, R. M., \& Taylor, G. J. (2006). Alexithymia and levels of processing: Evidence for an overall deficit in remembering emotion words. Journal of Research in Personality, 40, 713-733.

Lundh, L. G., Johnsson, A., Sundqvist, K., \& Olsson, H. (2002). Alexithymia, memory of emotion, emotional awareness, and perfectionism. Emotion, 2, 361-379.

Lundh L,G., \& Simonsson-Sarnecki, M. (2002) Alexithymia and cognitive bias for emotional information. Personality and Individual Differences 32, 1063-1075. 
Lundqvist, D., Flykt, A., \& Ohman, A. (1998). The Karolinska Directed Emotional Faces $(K D E F)$. Stockholm: Department of Neurosciences Karolinska Hospital.

Mason, O., Tyson, M., Jones, C., \& Potts, S. (2005). Alexithymia: its prevalence and correlates in a British undergraduate sample. Psychology and Psychotherapy: Theory, Research and Practice, 78(1), 113-125.

Mayer, J. D., McCormick, L. J., \& Strong, S. E. (1995). Mood-Congruent Memory and Natural Mood: New Evidence. Personality and Social Psychology Bulletin, 21(7),736-746.

McDonald, S., Flanagan, S., \& Rollins, J. (2002). The Awareness of Social Inference Test. Bury St. Edmonds, England: Thames Valley Test Company

McDonald, S., Flanagan, S., Martin, I., and Saunders, C. (2004). The ecological validity of TASIT: A test of social perception. Neuropsychological Rehabilitation, 2004. 14, 285-302.

McDonald, S., Bornhofen, C., Shum, D. Long, E., Saunders, C. \& Neulinger, K. (2006) Reliability and validity of The Awareness of Social Inference Test (TASIT): A clinical test of social perception, Disability and Rehabilitation, 28:24, 1529-1542, DOI: $\underline{10.1080 / 096382806006461}$

Meltzer, M. A., \& Nielson, K. A. (2010). Memory for emotionally provocative words in alexithymia: a role for stimulus relevance. Consciousness and cognition, 19(4), 1062-1068.

Nielson, K. A., \& Meltzer, M. A. (2009). Modulation of long-term memory by arousal in alexithymia: The role of interpretation. Consciousness and cognition, 18(3), 786-793.

Noreen, S., \& Ridout, N. (2010). Short-term memory for emotional faces in dysphoria. Memory, 18(5), 486-497.

Ochsner, K. N. (2000). Are aVective events richly recollected or simply familiar. The experience and process of recognizing feelings past. Journal of Experimental PsychologyGeneral, 129, 242-261.

Panayiotou, G., Panteli, M., \& Vlemincx, E. (2018). Processing Emotions in Alexithymia: A Systematic Review of Physiological Markers. Alexithymia: Advances in Research, Theory, and Clinical Practice, 291.

Parker, J. D., Taylor, G. J., \& Bagby, R. M. (2003). The 20-Item Toronto Alexithymia Scale: III. Reliability and factorial validity in a community population. Journal of psychosomatic research, 55(3), 269-275.

Parker, P. D., Prkachin, K. M., \& Prkachin, G. C. (2005). Processing of facial expressions of negative emotion in alexithymia: the influence of temporal constraint. Journal of personality, 73(4), 1087-1107.

Parks, T. E. (1966) Signal-detectability theory of recognition memory. Psychological Review, $73,44-58$

Pouga, L., Berthoz, S., de Gelder, B., \& Grezes, J. (2010). Individual differences in socioaffective skills influence the neural bases of fear processing: the case of alexithymia. Human brain mapping, 31(10), 1469-1481. 
Prkachin, G. C., Casey, C., \& Prkachin, K. M. (2009). Alexithymia and perception of facial expressions of emotion. Personality and Individual Differences, 46(4), 412-417.

Rajaram, S. (1998). The effects of conceptual salience and perceptual distinctiveness on conscious recollection. Psychonomic Bulletin Review, 5, 71-78.

Ridout, N., Astell, A. J., Reid, I. C., Glen, T. \& O' Carroll, R. E. (2003). Memory bias for emotional facial expressions in major depression Cognition and Emotion 17 (1) 101 - 122.

Ridout, N., O’Carroll, R. E., Dritschel, B., Christmas, D., Eljamel, M., \& Matthews, K. (2007). Emotion recognition from dynamic emotional displays following anterior cingulotomy and anterior capsulotomy for chronic depression. Neuropsychologia, 45(8), 1735-1743.

Ridout, N., Noreen, A., \& Johal, J. (2009a). Memory for emotional faces in naturally occurring dysphoria and induced negative mood. Behaviour, Research \& Therapy 47, 851860.

Ridout, N., Dritschel, B., Matthews, K. McVicar, M. Reid, I. C., \& O’ Carroll, R. E (2009b). Memory for emotional faces in major depression following judgment of physical facial characteristics at encoding. Cognition and Emotion. 23(4); 739-752.

Ridout, N., Thom, C. \& Wallis, D. J. (2010). Emotion recognition and alexithymia in females with non-clinical disordered eating. Eating Behaviors 11; 1-5.

Ridout, N., Wallis, D. J., Autwal, Y., \& Sellis, J. (2012). The influence of emotional intensity on facial emotion recognition in disordered eating. Appetite, 59(1), 181-186.

Russell, J. A. (1993). Forced-choice response format in the study of facial expression. Motivation and Emotion, 17,41-51.

Sharot, T. \& Phelps, E. A. (2004). How arousal modulates memory: disentangling the effects of attention and retention. Cognitive. Affective. Behavioural. Neuroscience. 4, 294-306.

Scarpazza, C. (2015). Deficit in the Emotional Embodiment in Alexithymia (Doctoral dissertation, alma).

Sharpe, E., Wallis, D. J., \& Ridout, N. (2016). The influence of variations in eating disorderrelated symptoms on processing of emotional faces in a non-clinical female sample: An eyetracking study. Psychiatry research, 240, 321-327.

Starita, F., Borhani, K., Bertini, C., \& Scarpazza, C. (2018). Alexithymia is related to the need for more emotional intensity to identify static fearful facial expressions. Frontiers in psychology, 9. 929doi: 10.3389/fpsyg.2018.00929

Stone, L. A., \& Nielson, K. A. (2001). Intact physiological response to arousal with impaired emotional recognition in alexithymia. Psychotherapy and psychosomatics, 70(2), 92-102.

Sullivan, L., Camic, P. M., \& Brown, J. S. (2015). Masculinity, alexithymia, and fear of intimacy as predictors of UK men's attitudes towards seeking professional psychological help. British journal of health psychology, 20(1), 194-211. 
Suslow, T., Kersting, A., \& Arolt, V. (2003). Alexithymia and incidental learning of emotional words. Psychological Reports, 93, 1003-1012.

Takahashi, J., Hirano, T., \& Gyoba, J (2015). Effects of facial expressions on visual shortterm memory in relation to alexithymia traits. Personality and Individual Differences, 83, $128-135$.

Talmi, D., Luk, B. T., McGarry, L. M., \& Moscovitch, M. (2007). The contribution of relatedness and distinctiveness to emotionally-enhanced memory. Journal of Memory and Language, 56(4), 555-574.

Talmi, D. (2013). Enhanced emotional memory: Cognitive and neural mechanisms. Current Directions in Psychological Science, 22(6), 430-436.

Tottenham, N., Tanaka, J. W., Leon, A. C., McCarry, T., Nurse, M. et al. (2009). The NimStim set of facial expressions: Judgments from untrained research participants. Psychiatry Research, 168 (3), 242-249.

Vermeulen, N., Luminet, O., \& Corneille, O. (2006). Alexithymia and the automatic processing of affective information: Evidence from the Affective Priming Paradigm. Cognition and Emotion, 20, 64-91.

Vermeulen, N., Luminet, O., Cordovil de Sousa, M., \& Campanella, S. (2008). Attentional processing of emotional facial expressions in alexithymia: A visual ERP study. Cognition and Emotion, 22, 1052-1067.

Vermeulen, N., \& Luminet, O. (2009). Alexithymia factors and memory performances for neutral and emotional words. Personality and Individual Differences, 47, 305-309.

Vermeulen, N., Toussaint, J. \& Luminet, O. (2010). The Influence of Alexithymia and Music on the Incidental Memory for Emotion Words. European Journal of Personality, 24, 551568.

Wallis, D. J., Ridout, N., \& Sharpe, E. (2018). The influence of non-clinical eating-related psychopathology on the recognition of emotion from static faces and realistic social interactions. Eating Behaviors, 29, 19-24.

Wechsler, D. (1981). Wechsler Adult Intelligence Scale- revised. Psychological Corporation.

Wells, T. T., Beevers, C. G., Robison, A. E., \& Ellis, A. J. (2010). Gaze behavior predicts memory bias for angry facial expressions in stable dysphoria. Emotion, 10(6), 894.

Williams, J. M. G., \& Broadbent, K. (1986). Autobiographical memory in attempted suicide patients. Journal of Abnormal Psychology, 95, $144-149$.

Zhang, L., Zhu, C., Ye, R., Cao, Z., et al. (2011). Impairment of conflict processing in alexithymic individuals. Neuroscience letters, 504(3), 261-264.

Zigmond, A.S. \& Snaith, R. P. (1983). The Hospital Anxiety and Depression Scale. Acta Psychriatica Scandinavia, 67, 361-370. 
Table 1: Mean age, depression, anxiety and alexithymia scores of the participants in study 1 (Standard deviations are presented in parentheses).

\begin{tabular}{lcc}
\hline $\mathrm{n}=39$ & Mean (SD) & Range \\
\hline Age & $19.50(1.1)$ & $18-22$ \\
Alexithymia (TAS-20) & $39.69(8.0)$ & $25-60$ \\
Difficulty identifying feelings (TAS-DIF) & $11.59(3.5)$ & $7-22$ \\
Difficulty describing feelings (TAS-DDF) & $10.97(4.4)$ & $5-21$ \\
Externally oriented thinking (TAS-EOT) & $17.23(3.3)$ & $8-24$ \\
Anxiety (HADS) & $5.64(3.0)$ & $0-11$ \\
Depression (HADS) & $2.08(1.8)$ & $0-7$ \\
\hline
\end{tabular}

TAS-20 = Toronto Alexithymia Scale; HADS = Hospital Anxiety and Depression Scale 
Table 2. Mean recognition memory performance indices (Study 1) as a function of emotional and neutral faces (standard deviations are presented in parentheses).

\begin{tabular}{lccc}
\hline & Emotional & Neutral & Significance \\
\hline Correct recognitions (max 10) & $6.22(1.4)$ & $5.97(2.1)$ & $\mathrm{t}(38)=.72, \mathrm{p}>.05$ \\
False positives (max 10) & $2.8(1.3)$ & $3.08(1.8)$ & $\mathrm{t}(38)=1.18, \mathrm{p}>.05$ \\
d' (sensitivity) & $1.04(.36)$ & $.86(.44)$ & $\mathrm{t}(38)=1.42, \mathrm{p}>.05$ \\
C (response bias) & $.15(.38)$ & $.14(.44)$ & $\mathrm{t}(38)=.14, \mathrm{p}>.05$ \\
Remember (max 10) & $3.12(1.4)$ & $2.77(2.1)$ & $\mathrm{T}(39)=1.35, \mathrm{p}>.05$ \\
Know (max 10) & $1.66(1.1)$ & $1.87(1.5)$ & $\mathrm{T}(39)=1.1, \mathrm{p}>.05$ \\
Guess (max 10) & $1.41(1.41)$ & $1.41(1.0)$ & $\mathrm{T}(39)=.29, \mathrm{p}>05$ \\
\hline
\end{tabular}

$\mathrm{T}=$ Wilcoxon tests and $\mathrm{t}=$ paired samples $\mathrm{t}$-tests 
Table 3. Hierarchical regression to predict d' and hits for emotional faces with alexithymia subscales as predictor variables (controlling for age and mood).

\begin{tabular}{|c|c|c|c|c|c|c|c|c|c|c|}
\hline \multirow{2}{*}{\multicolumn{2}{|c|}{$\mathrm{DV}=\mathrm{d}^{\prime}$ emotion }} & \multicolumn{4}{|c|}{ Model Summary } & \multicolumn{5}{|c|}{ Contribution of Each Factor at Step 2} \\
\hline & & $\mathrm{R}^{2}$ & $\mathrm{R}^{2} \Delta$ & $\mathrm{F}$ & $\mathrm{p}$ & B & SE & $\mathrm{b}$ & $\mathrm{t}$ & $\mathrm{p}$ \\
\hline \multirow[t]{4}{*}{ Step 1} & & .12 & - & 1.53 & .22 & & & & & \\
\hline & Age & & & & & -.04 & .05 & -.22 & -.78 & .44 \\
\hline & HADS-D & & & & & -.01 & .03 & -.04 & -.27 & .79 \\
\hline & HADS-A & & & & & -.03 & .02 & -.27 & -1.56 & .13 \\
\hline \multirow[t]{4}{*}{ Step 2} & & .44 & .32 & 5.93 & .003 & & & & & \\
\hline & DIF & & & & & .06 & .02 & .60 & 3.52 & .001 \\
\hline & DDF & & & & & -.04 & .02 & -.53 & -2.8 & .009 \\
\hline & EOT & & & & & -.01 & .02 & -.11 & -.63 & .53 \\
\hline \multirow{2}{*}{\multicolumn{2}{|c|}{$\begin{array}{l}\text { DV= Hits } \\
\text { emotion }\end{array}$}} & \multicolumn{4}{|c|}{ Model Summary } & \multicolumn{5}{|c|}{ Contribution of Each Factor at Step 2} \\
\hline & & & & & & & & & & \\
\hline & & $\mathrm{R}^{2}$ & $\mathrm{R}^{2} \Delta$ & $\mathrm{F}$ & $\mathrm{p}$ & B & $\mathrm{SE}$ & $\mathrm{b}$ & $\mathrm{t}$ & $\mathrm{p}$ \\
\hline \multirow[t]{4}{*}{ Step 1} & & .003 & - & .99 & & & & & & \\
\hline & Age & & & & & .002 & .02 & .02 & .08 & .93 \\
\hline & HADS-D & & & & & -.01 & .02 & -.13 & -.64 & .53 \\
\hline & HADS-A & & & & & .003 & .01 & .06 & .28 & .78 \\
\hline \multirow[t]{4}{*}{ Step 2} & & .13 & .13 & 1.5 & .23 & & & & & \\
\hline & DIF & & & & & .02 & .01 & .42 & 1.96 & .06 \\
\hline & DDF & & & & & -.01 & .01 & -.37 & -1.54 & .13 \\
\hline & EOT & & & & & .002 & .01 & .29 & .29 & .78 \\
\hline
\end{tabular}


Table 4: Mean age, mood and alexithymia scores of the participants in Study 2 (Standard deviation are presented in parentheses).

\begin{tabular}{lcc}
\hline $\mathrm{n}=38$ & Mean (SD) & Range \\
\hline Age & $19.63(2.7)$ & $18-34$ \\
Alexithymia (TAS-20) & $42.00(12.8)$ & $23-71$ \\
Difficulty identifying feelings (TAS-DIF) & $12.89(6.1)$ & $7-27$ \\
Difficulty describing feelings (TAS-DDF) & $11.58(5.3)$ & $5-23$ \\
Externally oriented thinking (TAS-EOT) & $17.26(4.1)$ & $11-30$ \\
Anxiety (HADS) & $6.97(3.1)$ & $3-14$ \\
Depression (HADS) & $3.05(2.3)$ & $0-9$ \\
\hline
\end{tabular}

TAS-20 $=$ Toronto Alexithymia Scale; HADS = Hospital Anxiety and Depression Scale. 
Table 5. Mean recognition memory performance indices (Study 2), as a function of emotional and neutral video clips (standard deviations are presented in parentheses).

\begin{tabular}{lccc}
\hline & Emotional & Neutral & Significance \\
\hline Correct recognitions $(\max 5)$ & $4.49(.58)$ & $4.79(.47)$ & $\mathrm{T}(38)=329.5, \mathrm{p}<.05$ \\
False positives $(\max 3)$ & $.27(.30)$ & $.11(.31)$ & $\mathrm{T}(38)=73.5, \mathrm{p}<.05$ \\
Remember $(\max 5)$ & $3.68(.96)$ & $4.13(1.1)$ & $\mathrm{T}(38)=455, \mathrm{p}<.01$ \\
Know (max 5) & $.75(.67)$ & $.55(1.0)$ & $\mathrm{T}(38)=319.5, \mathrm{p}>.05$ \\
\hline $\mathrm{T}=$ Wilcoxon tests & & &
\end{tabular}


Table 6. Hierarchical regression to predict correct emotion recognition of angry video clips with alexithymia subscales as predictor variables (controlling for age and mood).

\begin{tabular}{|c|c|c|c|c|c|c|c|c|c|c|}
\hline \multicolumn{2}{|c|}{$\mathrm{DV}=\mathrm{ER}$ angry } & \multicolumn{4}{|c|}{ Model Summary } & \multicolumn{5}{|c|}{ Contribution of Each Factor at Step 2} \\
\hline & & $\mathrm{R}^{2}$ & $\mathrm{R}^{2} \Delta$ & $\mathrm{F}$ & $\mathrm{p}$ & B & $\mathrm{SE}$ & $\mathrm{b}$ & $\mathrm{t}$ & $\mathrm{p}$ \\
\hline \multirow[t]{4}{*}{ Step 1} & & .06 & - & .66 & .58 & & & & & \\
\hline & Age & & & & & .27 & .41 & .12 & .66 & .51 \\
\hline & HADS-D & & & & & -.57 & .63 & -.21 & 1.0 & .37 \\
\hline & HADS-A & & & & & .4 & .37 & .12 & 1.1 & .30 \\
\hline \multirow[t]{4}{*}{ Step 2} & & .20 & .14 & 1.8 & .16 & & & & & \\
\hline & DIF & & & & & -.50 & .22 & -.49 & 2.3 & .031 \\
\hline & DDF & & & & & .39 & .31 & .33 & 1.3 & .22 \\
\hline & EOT & & & & & -.01 & .28 & -.1 & .04 & .97 \\
\hline
\end{tabular}


Table 7. Hierarchical regression to predict recognition memory 'hits' and remember scores for emotional video clips with alexithymia subscales as predictor variables (controlling for age and mood).

DV = Hits $\quad$ Model Summary $\quad$ Contribution of Each Factor at Step 2

emotion

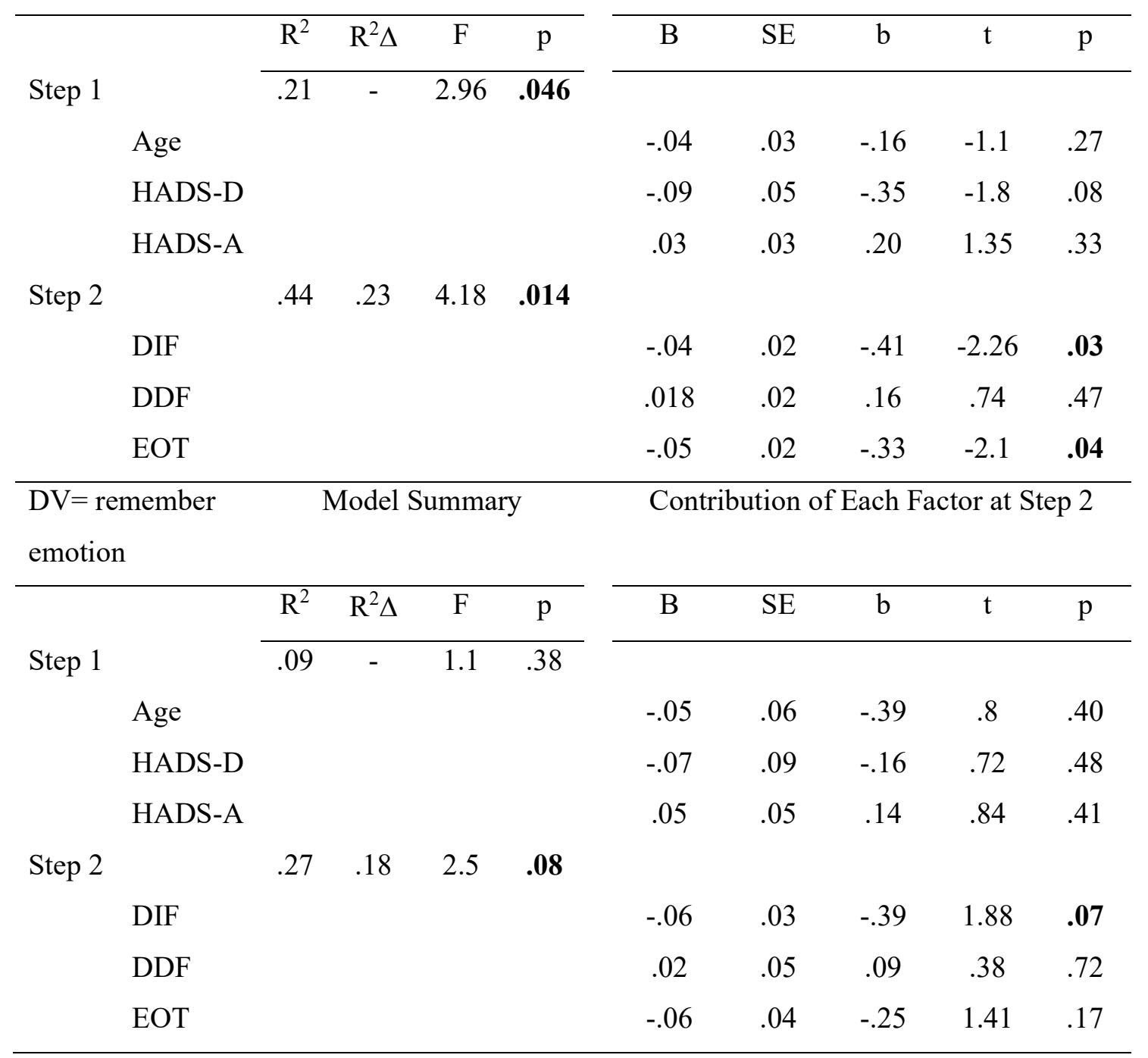


Table 8. Hierarchical regression to predict recognition memory know scores for neutral video clips with alexithymia subscales as predictor variables (controlling for age and mood).

\begin{tabular}{|c|c|c|c|c|c|c|c|c|c|c|}
\hline \multicolumn{2}{|c|}{$\mathrm{DV}=$ know neutral } & \multicolumn{4}{|c|}{ Model Summary } & \multicolumn{5}{|c|}{ Contribution of Each Factor at Step 2} \\
\hline & & $\mathrm{R}^{2}$ & $\mathrm{R}^{2} \Delta$ & $\mathrm{F}$ & $\mathrm{p}$ & $\mathrm{B}$ & SE & $\mathrm{b}$ & $\mathrm{t}$ & $\mathrm{p}$ \\
\hline \multirow[t]{4}{*}{ Step 1} & & .09 & - & 1.1 & .37 & & & & & \\
\hline & Age & & & & & -.07 & .06 & -.18 & 1.1 & .30 \\
\hline & HADS-D & & & & & .16 & .10 & .38 & 1.7 & .096 \\
\hline & HADS-A & & & & & -.06 & .06 & -.19 & 1.1 & .27 \\
\hline \multirow[t]{4}{*}{ Step 2} & & .29 & .21 & 3.0 & .043 & & & & & \\
\hline & DIF & & & & & .09 & .03 & .54 & 2.67 & .012 \\
\hline & DDF & & & & & -.08 & .05 & -.42 & 1.72 & .096 \\
\hline & EOT & & & & & -.04 & .04 & -.18 & 1.0 & .31 \\
\hline
\end{tabular}

